\title{
Autophagy Inhibition and Inflammation Activation Induced by Phosphorylated Alpha-synuclein Through Toll-like Receptor 2 Pathway in the Hippocampus of MPTP Mouse Model of Parkinson's Disease Contribute to Its Cognitive Function Decline in the Early Stage
}

Ke-Zhong Zhang ( $\sim$ kezhong_zhang1969@126.com )

Department of Neurology, The First Affiliated Hospital of Nanjing Medical University, Nanjing 210029 https://orcid.org/0000-0002-4437-1095

Jing Wu

Jiangsu Province Hospital and Nanjing Medical University First Affiliated Hospital

Wenwen Jiang

Jiangsu Province Hospital and Nanjing Medical University First Affiliated Hospital

Ye Wang

Jiangsu Province Hospital and Nanjing Medical University First Affiliated Hospital

Zhe Rong

Jiangsu Province Hospital and Nanjing Medical University First Affiliated Hospital

Li Sun

Suzhou Ninth People's Hospital

Yue Cheng

Jiangsu Province Hospital and Nanjing Medical University First Affiliated Hospital

\section{Research}

Keywords: Parkinson's disease, cognitive decline, phosphorylated alpha-synuclein, Toll-like receptor 2, autophagy, neuroinflammation

Posted Date: December 15th, 2020

DOl: https://doi.org/10.21203/rs.3.rs-126752/v1

License: (c) (i) This work is licensed under a Creative Commons Attribution 4.0 International License. Read Full License 


\section{Abstract}

Background: To evaluate consecutively the cognitive function of a chronic mouse model of Parkinson's disease (PD) induced by 1-methyl-4-phenyl-1, 2, 3, 6-tetrahydropyridine/probenecid (MPTP/p) in different modeling periods, and to study the characteristics of cognitive decline in PD and its molecular mechanism.

Methods: In this study, we used MPTP and probenecid to induce a chronic PD mouse model, the Y-maze test to evaluate the cognitive function of the model in different modeling periods, western blotting (WB) to quantify phosphorylated alpha-synuclein (p-a-syn), Toll-like receptors (TLRs), Nod-like receptors (NLRs), Choline acetyltransferase (ChAT), glial fibrillary acidic protein (GFAP), p62, Interleukin-1 $\beta$ (IL-1 $\beta$ ) and other related indicators, immunohistochemical staining $(\mathrm{IHC})$ to characterize the expression of $\mathrm{p}-\alpha-\mathrm{syn}$, immunofluorescence staining (IF) to observe the co-localization of p-a-syn and cholinergic neurons $\left(\mathrm{ChAT}^{+}\right)$or astroglias $\left(\mathrm{GFAP}^{+}\right)$in the hippocampus. The TLR2 inhibitor CU-CPT22 was used to intervene in the subacute MPTP PD mouse model and then the expression of TLR2, ChAT, GFAP, p62 and IL-1 $\beta$ were evaluated again.

Results: In the Y-maze test after $1 / 2 / 3 / 4 / 5$ weeks of administration, the percentage of bouts to the Novel arm and the percentage of duration in the Novel arm of the MPTP/p group mice were lower than those of the Saline group mice, and there were statistical differences at the second week. At the same time, the pa-syn monomer in the hippocampus of chronic MPTP/p PD mice increased, accompanied by the increase of GFAP, TLR2, Nuclear factor-KB (NF-KB), NLR pyrin domain containing 3 (NLRP3), p62 and IL-1 $\beta$, and the decrease of the ratio of LC3囚 to LC3I (LC3囚/I). The p-a-syn in the hippocampus increased, and co-localized significantly with GFAP and slightly with ChAT. TLR2 inhibitor CU-CPT22 can reduce the expression of GFAP, p62 and IL-1 $\beta$ in the subacute MPTP mouse model.

Conclusion: Autophagy inhibition and inflammation activation induced by p-a-syn through TLR2 pathway in the hippocampus of MPTP mouse model of Parkinson's disease contribute to its cognitive function decline in the early stage, indicating the potential of TLR2 as a therapeutic target for PD cognitive decline.

\section{Background}

Parkinson's disease (PD) is the second most common neurodegenerative disease after Alzheimer's disease (AD). In addition to typical motor symptoms such as resting tremor and bradykinesia, PD also often manifests as a variety of complex nonmotor symptoms (NMS) that affect the whole body, such as cognitive decline, sleep disorders, and autonomic dysfunction, etc. ${ }^{[1]}$. Among them, cognitive decline is one of the most common and important NMS, which may occur in the early stage of PD course, and seriously affects the quality of patients' life and brings economic burdens ${ }^{[2]}$. However, the research on the pathogenesis of PD cognitive decline is still shallow. 
The cause of cognitive decline in PD has not been fully elucidated, but the pathological damage of Lewy Body (LB) in the marginal and cortical parts including the hippocampus seems to be the main reason ${ }^{[3]}$. The PD pathological damage marker is the LB deposited in the midbrain dopaminergic (DA) neurons, and the Ser129 phosphorylated a-synuclein (p-a-syn) is its main pathological components. Under normal circumstances, $p$ - $a$-syn is only less than $4 \%$, and the content of p-a-syn in the LB of the PD patients' brain is as high as $90 \%{ }^{[4,5]}$. Based on this, we reasonably put forward the hypothesis: p-a-syn may play an important role in the pathological process of PD cognitive decline.

Studies have suggested neuroinflammation may be of great significance to PD cognitive decline and may become a new therapeutic target ${ }^{[6]}$. The current research on PD inflammation mechanism mainly focuses on the activation of glial cells and the release of inflammatory factors ${ }^{[7]}$. Glial cells constitute $40 \%$ of the total number of human brain cells, and astroglias constitute $20-40 \%$ of glial cells. Astroglias are essential for maintaining brain homeostasis and protecting neurons. However, under a variety of pathological conditions including PD, they will react and cause neuroinflammation and neurodegeneration $[8,9,10]$. Studies have found that there is a-syn deposition in astrocytes in autopsy of patients with sporadic PD ${ }^{[11]}$. The Toll-like receptors (TLRs) pathway is an important inflammation regulation pathway. In the PD course, TLRs can recognize misfolded or aggregated a-syn, lead to the

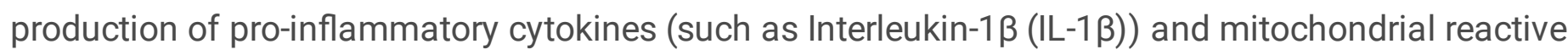
oxygen species (ROS), which are associated with increased levels of the autophagy marker p62 $[12,13,14]$. Autophagy is a cellular mechanism for the degradation and renewal of proteins and organelles in lysosomes. The neuroprotective effect of autophagy comes from its ability to eliminate the pathogenic form of a-syn, and autophagy dysfunction is one of the important pathogenesis of neurodegenerative diseases such as PD ${ }^{[15,16]}$. However, the correlation between factors such as p-a-syn, TLRs and PD cognitive decline remains unclear.

In this study, we found that chronic 1-methyl-4-phenyl-1, 2, 3, 6-tetrahydropyridine/probenecid (MPTP/p) PD model mice had early cognitive decline, and the $p$-a-syn monomer in the hippocampus increased, accompanied by the increase of glial fibrillary acidic protein (GFAP), TLR2, Nuclear factor-kB (NF-KB), Nod-like receptor pyrin domain containing 3 (NLRP3), p62 and IL-1 $\beta$, and the decrease of the ratio of LC3区 to LC3I (LC3区/I).The p-a-syn in the hippocampus increased, and co-localized significantly with GFAP, and slightly with Choline acetyltransferase (ChAT). TLR2 inhibitor CU-CPT22 can reduce the expression of GFAP, p62 and IL-1 $\beta$ in the hippocampus of the subacute MPTP PD mouse model, which proves that TLR2 inhibitor can reduce the autophagy inhibition and inflammation response induced by p-a-syn, indicating that TLR2 acts as the potential of therapeutic targets for PD cognitive decline.

\section{Materials And Methods}

\section{Animals and Treatments}

The experiment was performed on C57BL/ 6 mice (male, 8 weeks, $18-22 \mathrm{~g}$ ). These mice were obtained from the Qinglongshan Animal Breeding Farm (Nanjing, China), Jiangning District, Nanjing City, Jiangsu 
Province and kept in standard experiment room conditions. The mice were randomly housed in cages with a light/dark cycle of 12 hours (light on at 7:00 in the morning), a humidity of $60 \%$, and a temperature of $24 \pm 1^{\circ} \mathrm{C}$, and they could drink and eat freely. All procedures were strictly in accordance with the regulations and general recommendations of China's laboratory animal management regulations.

To establish a chronic MPTP/p PD mouse model: MPTP (20 mg/ $\mathrm{kg}$, dissolved in normal saline (NS), $0.1 \mathrm{ml} / 10 \mathrm{~g}$; Selleck, S4732) was injected subcutaneously into mice, and probenecid (250 mg $/ \mathrm{kg}$, dissolved in dimethyl sulfoxide (DMSO), 10 $\mathrm{l} / 10 \mathrm{~g}$; Macklin, P822732) was injected intraperitoneally one hour later to delay metabolism of MPTP, twice a week for 5 weeks. Control mice were treated with NS only. Part of the experimental group and control group mice were randomly subjected to behavior testing and sample collection within 7 days after $1 / 2 / 3 / 4 / 5$ weeks of administration.

Using TLR2 inhibitor CU-CPT22 to interfere in subacute MPTP PD mouse model: The mice were randomly divided into 4 groups: Saline group, MPTP group, CU-CPT22 group and MPTP+CU-CPT22 group. The saline group was injected with NS $(0.1 \mathrm{ml} / 10 \mathrm{~g})$ subcutaneously, the MPTP group was injected with MPTP (30 mg $/ \mathrm{kg}$, dissolved in NS, $0.15 \mathrm{ml} / 10 \mathrm{~g}$ ) subcutaneously, the CU-CPT22 group was injected

intraperitoneally with CU-CPT22 (3mg/kg, dissolved in DMSO, 40 $\mathrm{l} / 10 \mathrm{~g}$; Selleck, S8677) ${ }^{[17]}$ and in the CUCPT22+MPTP group, 30 minutes after intraperitoneal injection of CU-CPT22 (3mg/ $\mathrm{kg}$, dissolved in DMSO, $40 \mu \mathrm{l} / 10 \mathrm{~g})$, MPTP $(30 \mathrm{mg} / \mathrm{kg}$, dissolved in NS, $0.15 \mathrm{ml} / 10 \mathrm{~g})$ was injected subcutaneously. Once a day for 5 consecutive days. Sample collection was performed within 7 days after the last dose.

\section{Behavioral Analysis}

The Y-maze was a "Y"-shaped three-arm labyrinth made of polyvinyl chloride (PVC) board. The angle of each arm was 120 degrees. The size of each arm was $30 \mathrm{~cm} \times 8 \mathrm{~cm} \times 15 \mathrm{~cm}$ (length $\times$ width $\times$ height). Each arm had a movable partition in the center. The three arms were randomly defined as: Starting arm, Novel arm and Other arm. Each arm was marked with different geometric figures, allowing mice to form visual differences. The Y-maze experiment consisted of a training period and a test period, with an interval of 2 hours. During the training period, each Novel arm was blocked with a movable baffle with the same color, and the mouse was placed in the Starting arm with its head facing the wall and allowed to explore the Starting arm and the Other arm freely for 5 minutes. During the test period, the baffle was removed, the mouse was placed in the Starting arm with its head facing the wall, and the mouse was free to explore the three arms for 5 minutes. Before and after the experiment, the device was wiped with $75 \%$ ethanol to avoid the interference of residual odor to the experiment. A camera captured and recorded the movement of the mouse at 1.5 meters above the Y-maze. The analysis system (TopScanLite Version 2.00 Animal Routine Experimental Behavior Analysis System) connected to it can analyze the percentage of bouts and the percentage of duration the mouse entered the Novel arm. All tests were carried out by two independent experimenters who did not understand animal handling methods.

\section{Tissue Section Preparation}


The mice were anesthetized with sodium pentobarbital and perfused with NS and $4 \%$ paraformaldehyde (PFA) in sequence. The brains were harvested and fixed in 4\% PFA.

Paraffin section preparation: After dehydration, the tissue was embedded in paraffin and cut into $5 \mu \mathrm{m}$ coronal sections. Bake, dewax and rehydrate the paraffin slices.

Frozen section preparation: The tissue was dehydrated in $20 \%$ sucrose- phosphate buffer saline (PBS) and $30 \%$ sucrose-PBS and fixed with opti-mum cutting temperature (OCT) compound, and cut into $25 \mu \mathrm{m}$ coronal sections. The brain slices were immediately rinsed three times with $0.01 \mathrm{M}$ PBS.

\section{Immunohistochemistry Staining (IHC)}

Treat the slices with $3 \%$ hydrogen peroxide $\left(\mathrm{H}_{2} \mathrm{O}_{2}\right)$ and let them stand for 20 minutes in the dark at room temperature to quench the endogenous peroxidase activity. After washing with PBS, citrate buffer was used for antigen retrieval. The sections were blocked with PBST (PBS containing $0.3 \%$ Triton X-100) containing $5 \%$ bovine serum albumin (BSA) at room temperature for 1 hour. Subsequently, the sections were incubated overnight at $4^{\circ} \mathrm{C}$ with the following primary antibody: anti-p-a-syn (1:1,000; Abcam, ab51253). The sections were washed with PBS and incubated with the corresponding secondary antibody for 1 hour at room temperature: Peroxidase-Conjugated Goat Anti-Rabbit IgG $(H+L)$ (1:500; Yeason, 33101ES60). Rinse the samples and visualize the results by Diaminobenzidine (DAB) Chromogenic Kit (KeyGEN BioTECH, KGP1045) and Hematoxylin-Eosin Staining (Solarbio, G4070 and G1100). After dehydrating to make each part transparent, use neutral gum to mount the slide. Use Olympus BX51 microscope and MicroBrightField Stereo Investigator stereology system to observe and analyze images.

\section{Immunofluorescence Staining (IF)}

The tissue sections were blocked with PBST containing 5\% BSA for 1 hour at room temperature. Subsequently, the sections were incubated overnight at $4^{\circ} \mathrm{C}$ with the following primary antibodies: antiTyrosine hydroxylase (TH) (1:1,000; Sigma, T1299), anti-p-a-syn (1:1,000; Abcam, ab51253), anti-ChAT (1:200; Abcam, ab181023), anti-GFAP (1:500; Abcam, ab33922), anti-TLR2 (1:250; Abcam, ab209216), anti-p62 (1:250; Proteintech, 18420-1-AP) and anti-IL-1 3 (1:200; Santa Cruz, sc-52012). Wash the sections with PBS, then incubate with the corresponding secondary antibody for 1 hour at room temperature: Donkey anti-Mouse IgG $(\mathrm{H}+\mathrm{L})$ Highly Cross-Adsorbed Secondary Antibody, Alexa Fluor 488 (1:1,000; Invitrogen, A-21202) ), Goat anti-Mouse IgG ( $\mathrm{H}+\mathrm{L})$ Cross-Adsorbed Secondary Antibody, Alexa Fluor 555 (1:1,000; Invitrogen, A-21422), Goat anti-Rabbit IgG ( $\mathrm{H}+\mathrm{L})$ Cross-Adsorbed Secondary Antibody, Alexa Fluor 488 (1:1,000; Invitrogen, A-11008) and Donkey anti-Rabbit IgG $(\mathrm{H}+\mathrm{L})$ Highly Cross-Adsorbed Secondary Antibody, Alexa Fluor 555 (1:1,000; Invitrogen, A-31572). Use Hoechst (1:1,000; Cell Signaling Technology, 4082) to stain and mount the slides. Use ZEISS AXIO fluorescence microscope and MicroBrightField Stereo Investigator system to observe and analyze images.

\section{Western Blotting (WB)}


The mice were sacrificed after the behavioral test. The midbrain and hippocampus were quickly removed from the entire brain and cooled in liquid nitrogen. Tissue samples were homogenized in radio immunoprecipitation assay (RIPA) lysate (containing protease inhibitor and phosphatase inhibitor mixture), then centrifuged at $16,000 \mathrm{~g}$ at $4^{\circ} \mathrm{C}$ for 15 minutes, and the supernatant was collected. The protein concentration was determined by bicinchoninic acid (BCA) protein assay (Thermo Fisher, 23225). Use sodium dodecyl sulfate-polyacrylamide gel (SDS-PAGE, 8-12\%) electrophoresis to separate $30 \mu \mathrm{g}$ protein aliquots of each sample, and transfer them to polyvinylidene fluoride (PVDF) membrane (Millipore, ISEQ00010) by electrophoresis and transfer. Block with TBST (Tris buffered saline (TBS) containing Tween 20) containing 5\% skimmed milk powder for 2 hours. Then, the membrane was incubated overnight at $4^{\circ} \mathrm{C}$ with the following primary antibodies: anti-TH (1:5,000; Sigma, T1299), anti-asyn (1:2,000; Abcam, ab1903), anti-p-a-syn (1:2,000; Abcam, ab51253), anti-p-tau (1:500; Invitrogen, MN1020), anti-AB1-42 (1:800; Abcam, ab12276), anti-IL-1 $\beta$ (1:200; Santa Cruz, sc-52012), anti-NF-kB (1:800; Bioss, bs-0465R), anti-ChAT (1:2,000; Abcam, ab181023), anti-GFAP (1:2,000; Abcam, ab33922), anti-TLR1 (1:200; Santa Cruz, sc-514399), anti-TLR2 (1:1,000; Abcam, ab209216), anti-TLR3 (1:200; Santa Cruz, sc-32232 ), anti-TLR4 (1:200; Santa Cruz, sc-293072), anti-TLR7 (1:200; Santa Cruz, sc57463), anti-NALP1 (1:100; Santa Cruz, sc-390133), anti-AIM2 ( 1:200; Santa Cruz, sc-515514), antiNLRP3 (1:1,000; AdipoGen, AG-20B-0014-C100), anti-ChAT (1:2,000; Abcam, ab181023), anti-GFAP (1:2,000; Abcam, ab33922), anti-LC3 (1:1,000; Proteintech, 14600-1-AP), anti-p62 (1:1,000; Proteintech, 18420-1-AP) and anti-glyceraldehyde-3-phosphate dehydrogenase (GAPDH) (1:2,000; Proteintech, 600041-lg). Wash the membrane with TBST and incubate with the corresponding secondary antibody for 1 hour at room temperature: Rhodamine Red-X AffiniPure Goat Anti-Mouse $\lg G(H+L)(1: 5,000 ;$ Yeason, 33210ES60), Peroxidase-Conjugated Goat Anti -Rabbit lgG (H+L) (1:5,000; Yeason, 33101ES60). SuperSignal ${ }^{\text {TM }}$ West Femto Maximum Sensitivity Substrate kit (Thermo Fisher, 34096) and Tanon 5200 automatic chemiluminescence imaging analysis system were used for detection. Use ImageJ software (National Institutes of Health) to quantify the band intensity. The protein level was determined by normalization to the level of GAPDH and was presented relative to the control.

\section{Statistical Analysis}

Use GraphPad Prism 7 to analyze the results. The t-test was used for single factor analysis. In all studies, $P<0.05$ was considered statistically significant. The data graph was plotted as Mean \pm SD, and " $n$ " represents the number of samples in each group.

\section{Results}

\section{Chronic MPTP/p PD mouse model has early cognitive decline}

In this study, we performed a Y-maze test on MPTP/p mice in different modeling periods to evaluate their cognitive function (Fig. 1A). Among them, the percentage of bouts to the Novel arm (Bouts\%) represented the ability to explore novelty, and the percentage of duration in the Novel arm (Duration\%) represented the ability to explore space. The study found that after $1 / 2 / 3 / 4 / 5$ weeks of administration, the Bouts $\%$ and 
Duration\% of mice in the MPTP/p group were lower than those in the Saline group, and there were statistical differences at the second week (Bouts\%: $p<0.05$; Duration\%: $p<0.001$ ). It shows that the novelty exploration ability and spatial exploration ability of MPTP/p mice decrease in the second week. At the same time, we used IF (Fig. 1B) and WB (Fig. 1C) to detect DA neurons in the midbrain of mice treated with MPTP/p for 5 weeks (TH is a rate-limiting enzyme involved in DA synthesis, so $\mathrm{TH}^{+}$cells represent the number of DA neurons.) It was found that the number of $\mathrm{TH}^{+}$neurons in the midbrain of mice in the MPTP/p group was reduced by more than $50 \%$, and the difference was statistically significant (IF: $p<0.0001$; WB: $p<0.0001$ ), proving the successful establishment of the PD model. In summary, we believe that the chronic MPTP/p PD mouse model has early cognitive decline (see the discussion section for detailed analysis).

\section{In early PD mice's hippocampus, p-a-syn monomers increase with increased TLR2, inflammation and autophagy inhibition}

The protein expression levels whose abnormal aggregation may be related to cognitive decline in the hippocampal extracts of mice in the Saline group and the MPTP/p group of each week were detected (Fig. 2Ai). Compared with the control group, a-syn monomer (Fig. 2Aii) and p-tau (Fig. 2A囚) in the hippocampus of the MPTP/p mice increased in each week, and both were statistically significant; the p-asyn monomer (Fig. 2A囚) gradually increased with the prolonging of the modeling time, and the protein level detection difference in the 5th week was statistically significant, but the expression level of its aggregates (Fig. 2A囚, 2A囚) was not significantly different from that of the Saline group; the ratio of p-asyn monomer to a-syn monomer (Fig. 2A囚) showed an upward trend; $A \beta 1-42$ (Fig. 2A囚) increased in the 1 st week compared with the Saline group, but the difference was not statistically significant in the following weeks. In order to evaluate the inflammation level in the hippocampus of the MPTP/p group mice, we tested the expression levels of pro-IL-1 $\beta$, IL-1 $\beta$ and NF-KB (Fig. 2Bi). It was found compared with the Saline group, the levels of pro-IL-1 $\beta$, IL-1 $\beta$ (Fig. 2Bii), and NF-KB (Fig. 2B $\varangle$ ) of the MPTP/p group significantly increased. Therefore, we believe inflammation activation does exit in the hippocampus of the MPTP/p group mice.

In order to study the possible mechanism of inflammation activation, we quantitatively detected TLR1, TLR2, TLR3, TLR4, TLR7 of TLRs and NALP1, AIM2, and NLRP3 of NLRs (Fig. 2 Ci) in mouse hippocampus. It was found that compared with the Saline group, the expression of TLR1 (Fig. 2C区) of the MPTP/p group mice increased at the 1st and 3rd weeks; TLR2 (Fig. 2C区) gradually increased with the prolongation of the modeling time, and the difference in protein level detection at the 5 th week reached to statistical significance; TLR7 (Fig. 2C囚) increased in the 4th and 5th weeks; NALP1 (Fig. 2C囚) increased in the 1st week, and there was no statistically significant difference thereafter; NLRP3 (Fig. 2C区) had an upward trend with the prolonging of modeling time, and the difference in protein level detection at the 5th week was statistically significant. There were no significant differences in the other indicators during the 5-week test. At the same time, we analyzed the number of cholinergic neurons $\left(\mathrm{ChAT}^{+}\right)$and astrocytes $\left(\mathrm{GFAP}^{+}\right.$) in the hippocampus (Fig. 2Di), and found that compared with the Saline group, the MPTP/p 
group mice had more astrocytes (GFAP ${ }^{+}$, Fig. 2D』) in each week, and the difference was statistically significant; the number of cholinergic neurons (ChAT ${ }^{+}$, Fig. 2Dii) had a downward trend, but the difference was not statistically significant. In order to evaluate the autophagy level in the hippocampus of MPTP/p mice, we detected the expression levels of LC3 and p62 (Fig. 2Ei). It was found that compared with the Saline group, the LC3囚/I of the MPTP/p group mice decreased (Fig. 2Eii) and p62 (Fig. 2Eiii) increased. In summary, we believe that there is an increase in p-a-syn monomers in the hippocampus of chronic MPTP/p PD mice, accompanied by autophagy inhibition and inflammation activation, which may be related to the increased expression of TLR2 (see the discussion section for detailed analysis).

\section{In PD mice's hippocampus, p-a-syn increases and co-localizes significantly with GFAP and slightly with ChAT}

In order to further characterize and locate p-a-syn in the hippocampus of chronic MPTP/p PD mice, we applied IHC and IF to stain the tissue sections of the hippocampus of mice. It was found there was an increase in p-a-syn in the hippocampus of chronic MPTP/p PD mice in the 2nd week and the increase was more obvious in the 5th week (Fig. 3B), and it co-localized significantly with astrocytes (GFAP ${ }^{+}$) (Fig. 3Cii) and slightly with cholinergic neurons $\left(\mathrm{ChAT}^{+}\right)$(Fig. $3 \mathrm{Ci}$ ) (see the discussion section for detailed analysis).

\section{TLR2 inhibitor CU-CPT22 can reduce autophagy inhibition and inflammation response in subacute MPTP PD mouse}

The TLR2 inhibitor CU-CPT22 was used to intervene in the subacute MPTP PD mouse model, and IF was used to semi-quantitatively analyze the TLR2, ChAT, GFAP, p62 and IL-1 $\beta$ of the mouse hippocampus (Fig. 4A-E). We found that the TLR2 inhibitor CU-CPT22 can reduce the increased expression of TLR2, GFAP, p62 and IL-1 $\beta$ in the subacute MPTP PD mouse model. These three indicators GFAP, p62 and IL-1 $\beta$ showed no significant difference between the CU-CPT22 group and the Saline group, so the influence of CU-CPT22 on these three indicators was excluded. ChAT showed no difference among the four groups. In summary, we believe that inhibition of TLR2 can reduce the autophagy inhibition and inflammation response induced by $\mathrm{p}$-a-syn (see the discussion section for detailed analysis).

\section{Discussion}

According to reports, PD patients can experience cognitive decline in the early stage of the disease process ${ }^{[2]}$. The progressive PD mice induced by MPTP/p is a recognized classic model for the study of PD. It can reproduce the main neurochemical and anatomical pathologies of PD ${ }^{[18,19]}$. However, the cognitive function of this model is rarely reported. Therefore, in this study, we performed a Y-maze test on MPTP/p mice in different modeling periods to evaluate the cognitive function, and found the cognitive function of the MPTP/p group mice decreased after two weeks of administration significantly. In the following $3 / 4 / 5$ weeks, although the cognitive ability of the MPTP/p group mice was better than that of the MPTP/p group in the 2nd week, it was still worse than the Saline group in the same period (Fig. 1A). 
Therefore, we temporarily believe that the pathological process of cognitive decline is still in progress, and the improvement of cognitive ability in the MPTP/p group needs further research to explain this change, which may be affected by the model's own compensation and sample size. Or continuing to extend the administration time may cause more serious cognitive decline. Therefore, we currently believe that the 2nd week may be the critical time point for the change of cognitive function in the chronic MPTP/p PD model. It is necessary to further explore its pathological mechanism.

Some studies believe that protein misfolding in hippocampus and limbic system is one of the possible mechanisms of PD cognitive decline, which mainly involves a-syn, amyloid and tau ${ }^{[2]}$. $a$-syn is an important part of LB which is hallmarks of the histopathology of PD, and further studies have shown the hyperphosphorylation of Ser129 of a-syn may affect its solubility and membrane binding properties, thereby making it to be in a pathogenic state ${ }^{[20]}$. At the same time, studies have found that lower A $1-42$ baseline concentrations in cerebrospinal fluid (CSF) are related to poor cognitive ability, and may predict cognitive decline in PD patients ${ }^{[21]}$. And the pathology of tau has a good correlation with cognitive ability. The neurofibrillary tangles (NFT) composed of hyperphosphorylated tau is a common pathological feature of tau protein diseases ${ }^{[22]}$. Therefore, in this study, we detected quantitatively the level of a-syn, $p$ a-syn, p-tau and AB1-42 in hippocampal extracts by WB. In the 2 nd week, the indicators with statistical difference between the two groups were: $\alpha$-syn monomer (increased, $p<0.01$ ) and $p$-tau (increased, $p<$ $0.05)$; the indicator with difference that had a statistically significant trend (statistical differences appeared after the 2 nd week) between the two groups was p-a-syn monomer (increased). Compared with the control group, the a-syn monomer and p-tau in the hippocampus of chronic MPTP/p PD mice increased in each week. Among them, the increase in p-tau may be caused by the increase in a-syn [23]. The p-a-syn monomer gradually increased with the prolonged modeling time and the ratio of $p$-a-syn monomer to a-syn monomer also showed an upward trend, indicating p-a-syn may occupy an important position in the pathological process of the model. But there was no significant difference in the expression level of $p$-a-syn aggregates between two groups. This may be due to the limitations of the MPTP/p model, or the expression level of aggregates may increase if the modeling time continues to be extended. A $\beta 1-42$ increased in the 1st week, but the difference was not obvious in the following weeks, which was a transient increase (Fig. 2A). In summary, based on the reported results before and the results of this study, we currently believe that compared with A $\mathrm{A} 1-42$ and p-tau, abnormal p-a-syn expression in hippocampus is more likely to occupy a key position in the pathological process of PD cognitive decline in the early stage.

More and more evidences show that neuroinflammation is of great significance to PD cognitive decline [6]. The inflammatory response around the hippocampus can cause pro-IL-1 $\beta$ to activate into biologically active IL-1 $\beta$, which can promote the production of other cytokines (such as Tumor necrosis factor (TNF- $\alpha$ ) and IL-6), thereby affecting hippocampal neural activity and cognitive function ${ }^{[24,25]}{ }^{\text {. Induction of }}$ abnormal a-syn and mediation of TLRs and NLRs can cause the activation of IL-1 $\beta$. TLRs and NLRs are both families of transmembrane pattern recognition receptor (PRR) proteins and considered to be effective treatment strategies to slow down the progression of PD ${ }^{[13,26,27]}$. The secretion of IL-1 $\beta$ will 
also be regulated by autophagy ${ }^{[28]}$. In addition, increasing studies have shown astrocyte dysfunction is an important part of PD pathological process, and the lack of cholinergic neurotransmitters is related to the decline of PD cognitive ability ${ }^{[2,29]}$. Therefore, in this study, we used WB to quantitatively analyze the following indicators in the hippocampus extract : pro-IL-1 $\beta$, IL-1 $\beta$, NF-KB, TLRs (TLR1, TLR2, TLR3, TLR4 and TLR7), NLRs (NALP1, AIM2 and NLRP3), GFAP (biomarker of astrocytes), ChAT (biomarker of cholinergic neurons), autophagy-related index microtubule-associated protein 1 light chain $3 \otimes$ to I (LC3 $₫ /$ 『) and the LC3 binding adaptor protein p62. It was found in the $2 \mathrm{nd}$ week, the indicators with statistical difference between the two groups were: pro-IL-1 $\beta$ (increased, $p<0.001$ ), IL-1 $\beta$ (increased, $p<0.001$ ), NFKB (increased, $p<0.001$ ), GFAP (increased, $p<0.0001$ ), LC3 $/ / \mathbb{\text { (decreased, }} p<0.001$ ) and p62 (increased, $p<0.05$ ). The indicators with difference that had statistically significant trends (statistical differences appeared after the 2nd week) between the two groups were: TLR1 (increased), TLR2 (increased), TLR7 (increased) and NLRP3 (increased) (Fig. 2). The above results show that there is indeed inflammation activation and autophagy inhibition in the hippocampus of MPTP PD mouse model. In particular, in many PD animal models induced by overexpression of a-syn or MPTP, it has been consistently reported that TLR2 and TLR4 are up-regulated, and increasing evidences further link these two receptors with the pathogenesis of PD. Although there may be other TLRs involved in PD, there is little evidence. Therefore, this research focuses on the intrinsic correlation between TLR2 expression and cognitive decline in PD [26]. And NLRP3 showed an upward trend with the prolonging of the modeling time. This indicates that NLRP3 is more likely to be involved in the pathological process of cognitive disorder in PD. Combining the results of the reported research and this research, we propose the following hypothesis diagram (Fig. 5): Factors such as MPTP can cause abnormal deposition of p-a-syn in the hippocampus of patients with early PD, which may be related to autophagy inhibition. TLR2 in astroglias can recognize $p$-a-syn, activate the NF-KB pathway, and produce IL-1 $\beta$ and other inflammatory factors precursors. At the same time, p-asyn activates the NLRP3 inflammasome. After activation of the NLRP3 inflammasome, it then activates Cysteinyl aspartate specific proteinase 1 (Caspase-1), which can then cleave the IL-1 $\beta$ precursor and activate Gasdermin D. Gasdermin D will be transported to the cell membrane and form pores, so that astroglias can release the biologically active inflammatory factor IL-1 $\beta$ and trigger cell death $[12,13,30]$. This leads to the decline of cognitive function in early PD patients. But this hypothesis needs further research to verify.

In order to further characterize and locate p-a-syn in the hippocampus of MPTP/p PD model mice, we used IHC and IF to detect tissue sections in the hippocampus of the Saline group, MPTP/p-2W group and MPTP/p-5W group mice. The results showed p-a-syn increased in the hippocampus of the MPTP/p-2W group and the increase was more obvious in the 5th week (Fig. 3B). And p-a-syn co-localized significantly with GFAP, and slightly with ChAT (Fig. 3C), further suggesting that astrocytes may participate in the pathological process of PD cognitive function decline. In order to verify the role of TLR2 in the pathological process of MPTP-induced PD, we used the TLR2 inhibitor CU-CPT22 to intervene in the subacute MPTP PD mouse model, and applied IF to semi-quantitative analysis of TLR2, ChAT, GFAP, p62 and IL-1 $\beta$ in mouse hippocampus. The results showed that the TLR2 inhibitor CU-CPT22 can reduce the increased expression of GFAP, p62 and IL-1 $\beta$ in the subacute MPTP mouse model (Fig. 4). Combined with 
the above research results, we currently believe that inhibition of TLR2 can reduce the autophagy inhibition and inflammatory response induced by p-a-syn.

In summary, in this study, we found the chronic MPTP/p PD mouse model had early cognitive decline, the expression of $p$-a-syn in the hippocampus increased, and it co-localized with astroglias significantly. TLR2 inhibitors can reduce the autophagy inhibition and inflammation response. So we consider that autophagy inhibition and inflammation activation induced by p-a-syn through TLR2 pathway in the hippocampus of MPTP mouse model of Parkinson's disease contribute to its cognitive function decline in the early stage, indicating the potential of TLR2 as a therapeutic target for PD cognitive decline. But this discovery needs further research to consolidate.

\section{Conclusion}

Autophagy inhibition and inflammation activation induced by p-a-syn through TLR2 pathway in the hippocampus of MPTP mouse model of Parkinson's disease contribute to its cognitive function decline in the early stage, indicating the potential of TLR2 as a therapeutic target for PD cognitive decline.

\section{Abbreviations}

PD: Parkinson's disease

AD: Alzheimer's disease

NMS: nonmotor symptom

LB: Lewy Body

DA: dopaminergic

p-a-syn: phosphorylated a-synuclein

TLR: Toll-like receptor

IL-1 $\beta$ : Interleukin-1 $\beta$

ROS: reactive oxygen species

MPTP: 1-methyl-4-phenyl-1, 2, 3, 6-tetrahydropyridine

p: probenecid

GFAP: glial fibrillary acidic protein

NF-kB: Nuclear factor-kB 
NLRP3: Nod-like receptor pyrin domain containing 3

ChAT: Choline acetyltransferase

NS: normal saline

DMSO: dimethyl sulfoxide

PVC: polyvinyl chloride

PFA: paraformaldehyde

PBS: phosphate buffer saline

OCT: opti-mum cutting temperature

IHC: Immunohistochemistry

BSA: bovine serum albumin

DAB: Diaminobenzidine

IF: Immunofluorescence

TH: Tyrosine hydroxylase

WB: Western Blotting

RIPA: radio immunoprecipitation assay

BCA: bicinchoninic acid

SDS-PAGE: sulfate-polyacrylamide gel

PVDF: polyvinylidene fluoride

TBS: Tris buffered saline

GAPDH: glyceraldehyde-3-phosphate dehydrogenase

DG: denate gyrus

CSF: cerebrospinal fluid

NFT: neurofibrillary tangle

TNF-a: Tumor necrosis factor 
PRR: pattern recognition receptor

Caspase-1: Cysteinyl aspartate specific proteinase 1

\section{Declarations}

\section{Ethics approval and consent to participate}

All procedures were strictly in accordance with the regulations and general recommendations of China's laboratory animal management regulations.

\section{Consent for publication}

Not applicable.

\section{Availability of data and materials}

The datasets used and/or analyzed during the present study are available from the corresponding author upon reasonable request.

\section{Competing interests}

The authors declare that they have no competing interests.

\section{Funding}

This work was supported by grants from the National Natural Science Foundation of China (81671258 and 82071431) to Dr. Kezhong Zhang.

\section{Acknowledgements}

Not applicable.

\section{Authors' contributions}

Jing Wu, Wenwen Jiang and Ye Wang contributed equally to this work. All authors read and approved the final manuscript.

\section{References}

1. Schapira AHV, Chaudhuri KR, Jenner P. Non-motor features of Parkinson disease. Nat Rev Neurosci. 2017;18(7):435-450.

2. Aarsland D, Creese B, Politis M, et al. Cognitive decline in Parkinson disease. Nat Rev Neurol. 2017;13(4):217-231. 
3. Li Y, Sun H, Chen Z, Xu H, Bu G, Zheng H. Implications of GABAergic Neurotransmission in Alzheimer's Disease. Front Aging Neurosci. 2016;8:31.

4. Fujiwara $\mathrm{H}$, Hasegawa $\mathrm{M}$, Dohmae $\mathrm{N}$, et al. alpha-Synuclein is phosphorylated in synucleinopathy lesions. Nat Cell Biol. 2002;4(2):160-164.

5. Oueslati A. Implication of Alpha-Synuclein Phosphorylation at S129 in Synucleinopathies: What Have We Learned in the Last Decade? J Parkinsons Dis. 2016;6(1):39-51.

6. Rocha NP, Teixeira AL, Scalzo PL, et al. Plasma levels of soluble tumor necrosis factor receptors are associated with cognitive performance in Parkinson's disease. Mov Disord. 2014;29(4):527-531.

7. Deleidi M, Gasser T. The role of inflammation in sporadic and familial Parkinson's disease. Cell Mol Life Sci. 2013;70(22):4259-4273.

8. Verkhratsky A, Nedergaard M. Physiology of Astroglia. Physiol Rev. 2018;98(1):239-389.

9. von Bartheld CS, Bahney J, Herculano-Houzel S. The search for true numbers of neurons and glial cells in the human brain: A review of 150 years of cell counting. J Comp Neurol. 2016;524(18):38653895.

10. Arranz AM, De Strooper B. The role of astroglia in Alzheimer's disease: pathophysiology and clinical implications. Lancet Neurol. 2019;18(4):406-414.

11. Braak H, Sastre M, Del Tredici K. Development of alpha-synuclein immunoreactive astrocytes in the forebrain parallels stages of intraneuronal pathology in sporadic Parkinson's disease. Acta Neuropathol. 2007;114(3):231-241.

12. Daniele SG, Béraud D, Davenport C, Cheng K, Yin H, Maguire-Zeiss KA. Activation of MyD88dependent TLR1/2 signaling by misfolded a-synuclein, a protein linked to neurodegenerative disorders. Sci Signal. 2015;8(376):ra45.

13. Heneka MT, McManus RM, Latz E. Inflammasome signalling in brain function and neurodegenerative disease. Nat Rev Neurosci. 2018;19(10):610-621.

14. Dzamko N, Gysbers A, Perera G, et al. Toll-like receptor 2 is increased in neurons in Parkinson's disease brain and may contribute to alpha-synuclein pathology. Acta Neuropathol. 2017;133(2):303319.

15. Scrivo A, Bourdenx M, Pampliega O, Cuervo AM. Selective autophagy as a potential therapeutic target for neurodegenerative disorders. Lancet Neurol. 2018;17(9):802-815.

16. Kim C, Rockenstein E, Spencer B, et al. Antagonizing Neuronal Toll-like Receptor 2 Prevents Synucleinopathy by Activating Autophagy. Cell Rep. 2015;13(4):771-782.

17. Ohno K, Kuno A, Murase H, et al. Diabetes increases the susceptibility to acute kidney injury after myocardial infarction through augmented activation of renal Toll-like receptors in rats. Am J Physiol Heart Circ Physiol. 2017;313(6):H1130-h1142.

18. Yang W, Hao W, Meng Z, et al. Molecular Regulatory Mechanism and Toxicology of Neurodegenerative Processes in MPTP/Probenecid-Induced Progressive Parkinson's Disease Mice Model Revealed by Transcriptome. Mol Neurobiol. 2020. 
19. Porras G, Li Q, Bezard E. Modeling Parkinson's disease in primates: The MPTP model. Cold Spring Harb Perspect Med. 2012;2(3):a009308.

20. Zhou J, Broe M, Huang $Y$, et al. Changes in the solubility and phosphorylation of a-synuclein over the course of Parkinson's disease. Acta Neuropathol. 2011;121(6):695-704.

21. Parnetti L, Gaetani L, Eusebi P, et al. CSF and blood biomarkers for Parkinson's disease. Lancet Neurol. 2019;18(6):573-586.

22. Wang Y, Mandelkow E. Tau in physiology and pathology. Nat Rev Neurosci. 2016;17(1):5-21.

23. Duka T, Rusnak M, Drolet RE, et al. Alpha-synuclein induces hyperphosphorylation of Tau in the MPTP model of parkinsonism. Faseb j. 2006;20(13):2302-2312.

24. Bartsch T, Wulff P. The hippocampus in aging and disease: From plasticity to vulnerability. Neuroscience. 2015;309:1-16.

25. Hueston $\mathrm{CM}, \mathrm{O}$ 'Leary JD, Hoban AE, et al. Chronic interleukin-1 $\beta$ in the dorsal hippocampus impairs behavioural pattern separation. Brain Behav Immun. 2018;74:252-264.

26. Kouli A, Horne CB, Williams-Gray $\mathrm{CH}$. Toll-like receptors and their therapeutic potential in Parkinson's disease and a-synucleinopathies. Brain Behav Immun. 2019;81:41-51.

27. Han X, Sun S, Sun Y, et al. Small molecule-driven NLRP3 inflammation inhibition via interplay between ubiquitination and autophagy: implications for Parkinson disease. Autophagy. 2019;15(11):1860-1881.

28. Harris J, Lang T, Thomas JPW, Sukkar MB, Nabar NR, Kehrl JH. Autophagy and inflammasomes. Mol Immunol. 2017;86:10-15.

29. Booth HDE, Hirst WD, Wade-Martins R. The Role of Astrocyte Dysfunction in Parkinson's Disease Pathogenesis. Trends Neurosci. 2017;40(6):358-370.

30. Broz P, Dixit VM. Inflammasomes: mechanism of assembly, regulation and signalling. Nat Rev Immunol. 2016;16(7):407-420.

\section{Figures}




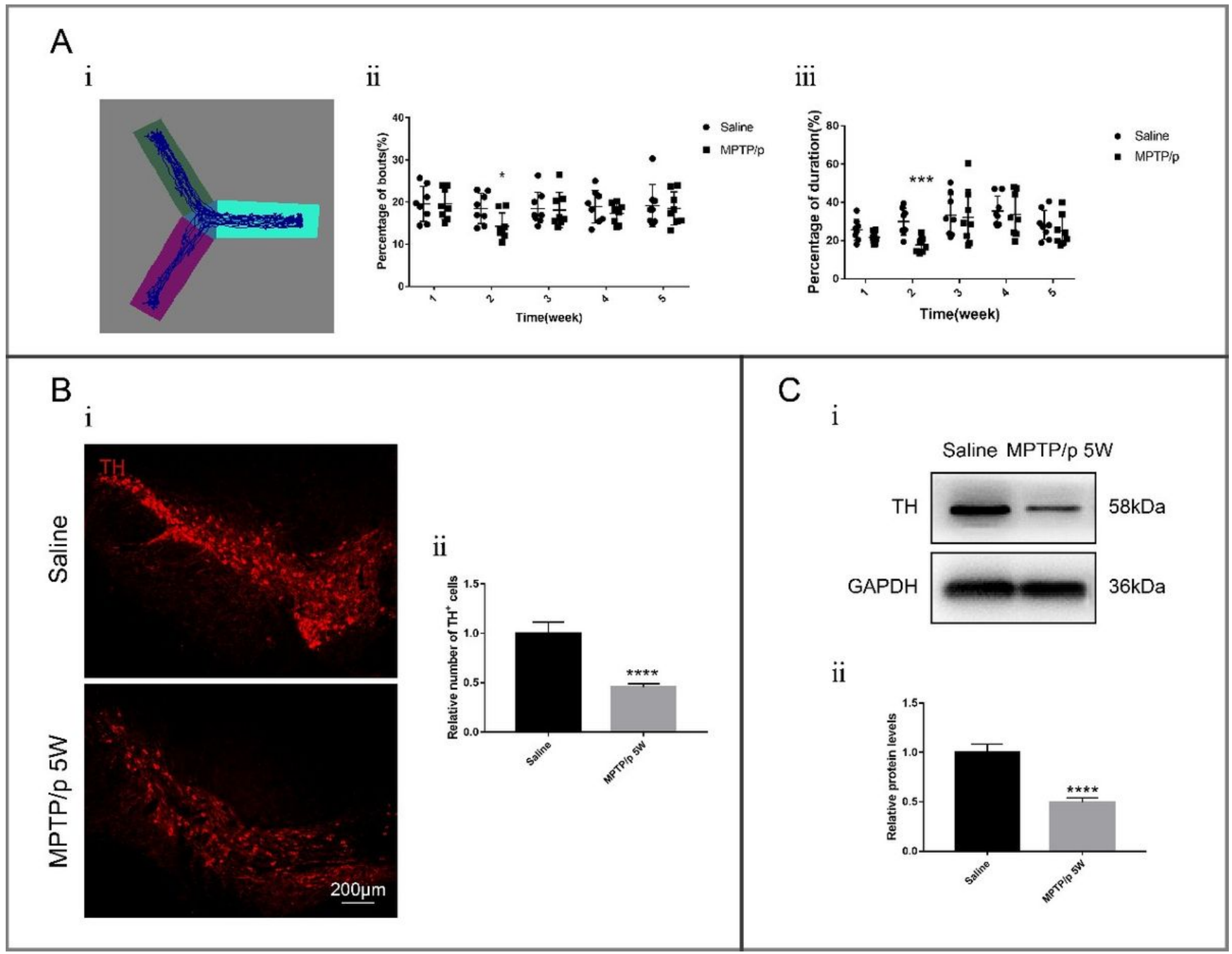

\section{Figure 1}

A: i: schematic diagram of Y-maze device. The blue line is the movement trajectory of the mice during the detection period; ii: analysis of the bouts\% to the Novel arm in the Y-maze test of the Saline group and

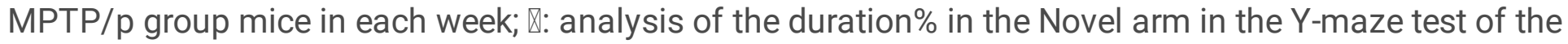
Saline group and MPTP/p group mice in each week. The data graph is plotted as Mean $\pm S D, n=8$. The statistical analysis method is t-test, and * means $p<0.05$ compared with Saline group; ${ }^{* \star \star}$ means $p<0.001$ compared with Saline group; B: i: TH IF of midbrain in the Saline group and MPTP/p-5W group mice; ii: quantitative analysis of the number of DA neurons $(\mathrm{TH}+)$. As shown in the scale bar, the appropriate magnification is selected. The data represents 6 independent experiments $(n=6)$, and the data graph is

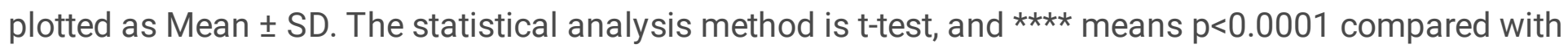
the Saline group; C: i: WB bands of TH in midbrain extracts of mice in the Saline group and MPTP/p-5W group. An additional movie file shows this in more detail; ii: quantitative analysis of the number of DA neurons $(\mathrm{TH}+)$. The data represents 6 independent experiments $(n=6)$, and the data graph is plotted as 


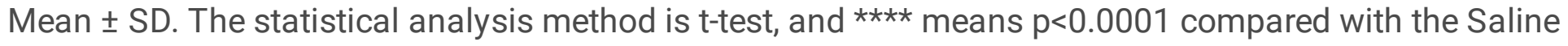
group.

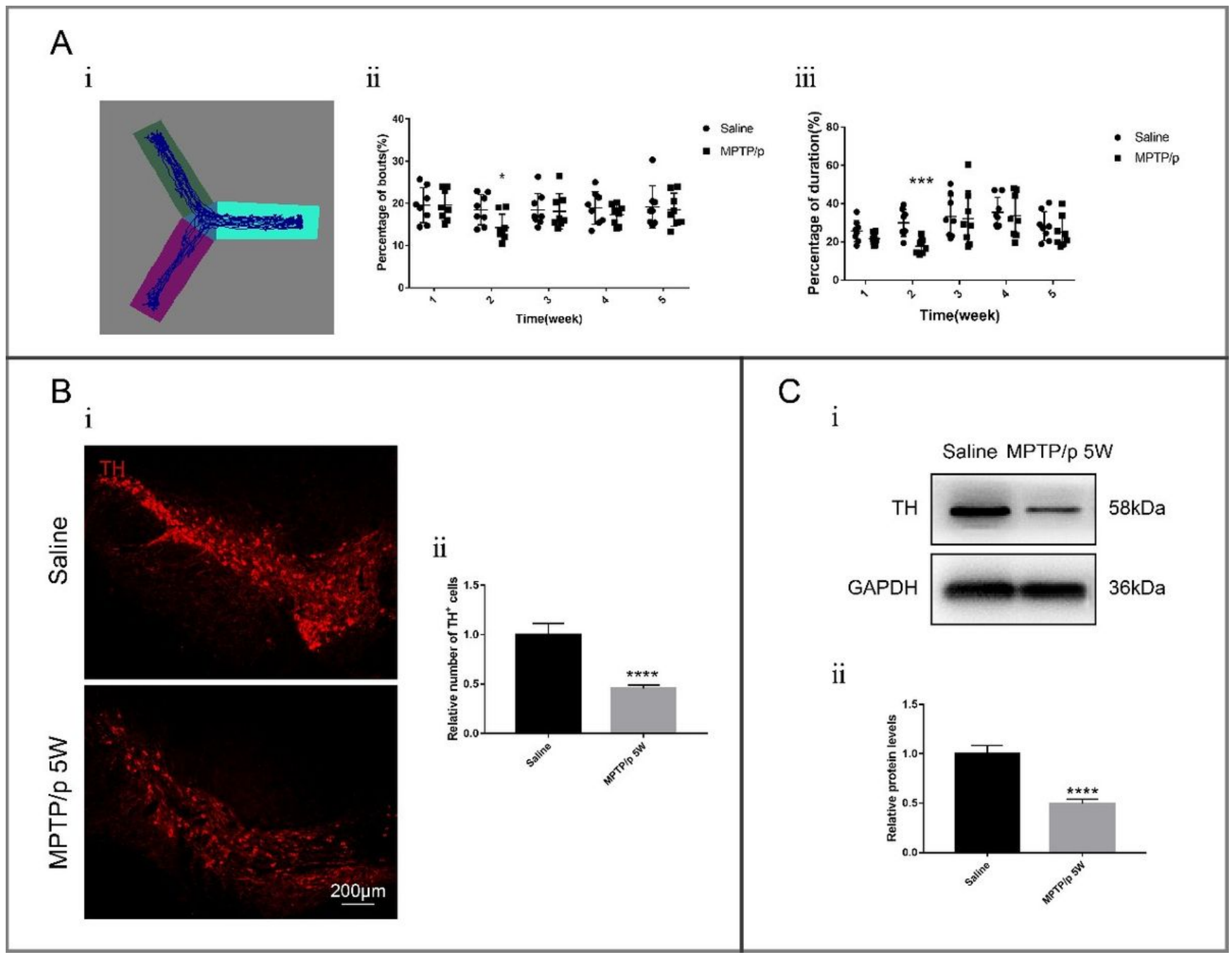

Figure 1

A: i: schematic diagram of Y-maze device. The blue line is the movement trajectory of the mice during the detection period; ii: analysis of the bouts\% to the Novel arm in the Y-maze test of the Saline group and MPTP/p group mice in each week; $\rrbracket$ : analysis of the duration\% in the Novel arm in the Y-maze test of the Saline group and MPTP/p group mice in each week. The data graph is plotted as Mean $\pm S D, n=8$. The statistical analysis method is t-test, and * means $p<0.05$ compared with Saline group; *** means $p<0.001$ compared with Saline group; B: i: TH IF of midbrain in the Saline group and MPTP/p-5W group mice; ii: quantitative analysis of the number of DA neurons $(\mathrm{TH}+)$. As shown in the scale bar, the appropriate magnification is selected. The data represents 6 independent experiments $(n=6)$, and the data graph is plotted as Mean \pm SD. The statistical analysis method is t-test, and $\star \star \star \star$ means $p<0.0001$ compared with the Saline group; C: i: WB bands of TH in midbrain extracts of mice in the Saline group and MPTP/p-5W group. An additional movie file shows this in more detail; ii: quantitative analysis of the number of DA 
neurons $(\mathrm{TH}+)$. The data represents 6 independent experiments $(n=6)$, and the data graph is plotted as

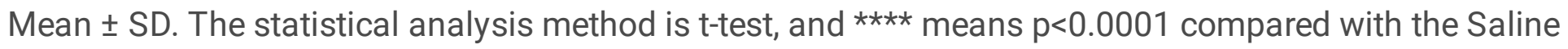
group.

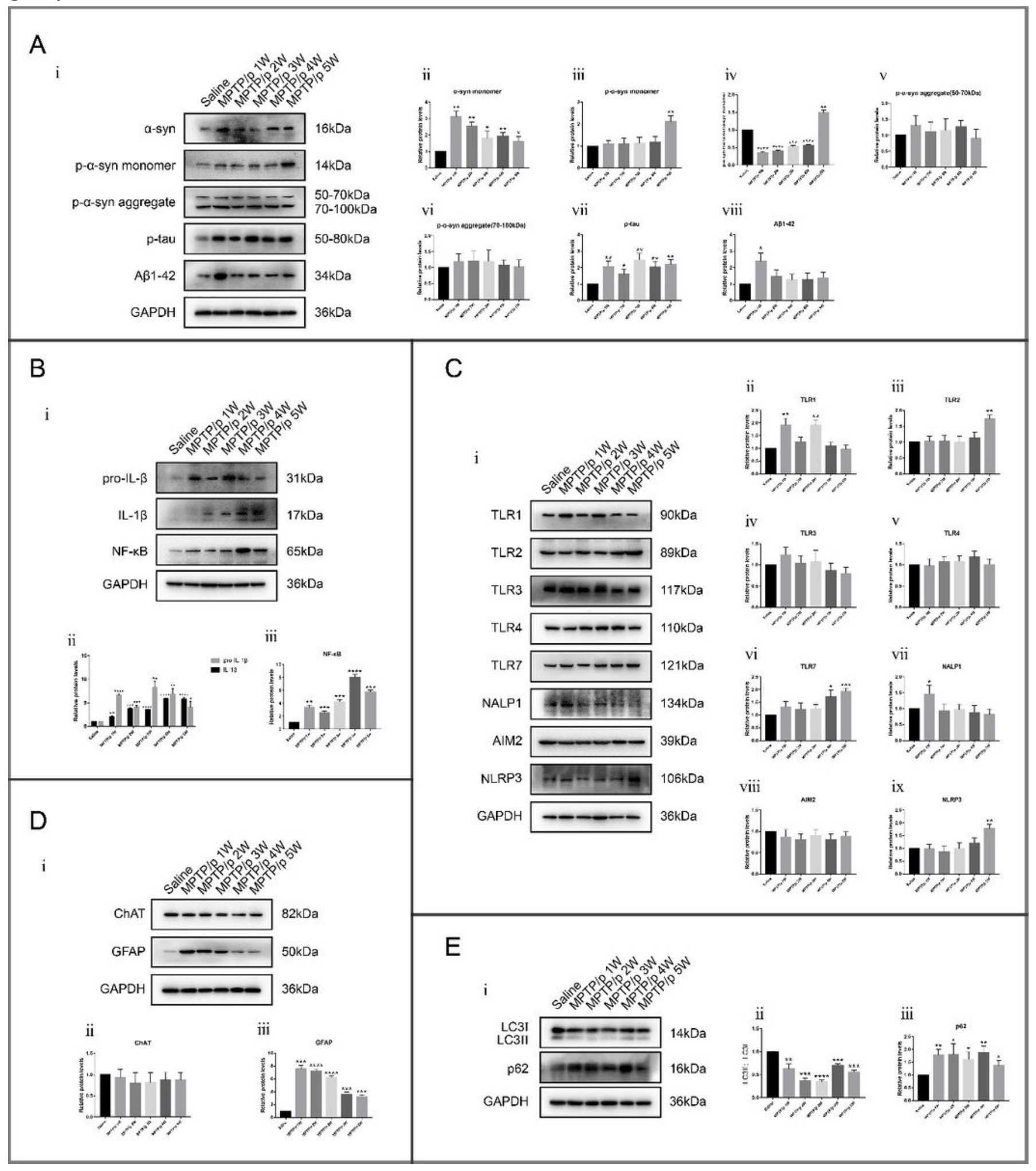

Figure 2

WB results of various indicators in the hippocampal extracts of mice in the Saline group and MPTP/p groups in each week. i: WB band of each index. An additional movie file shows this in more detail; ii-『: 
quantitative analysis of the protein expression level of the corresponding index in i. Use GAPDH as an internal reference. The data graph is plotted as Mean $\pm S D, n=4$. The statistical analysis method is t-test. * means $p<0.05$ compared with Saline group; ** means $p<0.01$ compared with Saline group; *** means $p<0.001$ compared with Saline group; and ${ }^{* \star \star *}$ means $p<0.0001$ compared with Saline group. A: a-syn monomer, p-a-syn monomer and aggregate, $p$-tau and A $31-42$; B: pro-IL-1 $\beta$, IL-1 $\beta$ and NF-KB; C: TLR1, TLR2, TLR3, TLR4, TLR7, NALP1, AIM2 and NLRP3; D: ChAT and GFAP; E: LC3区, LC3\ and p62.

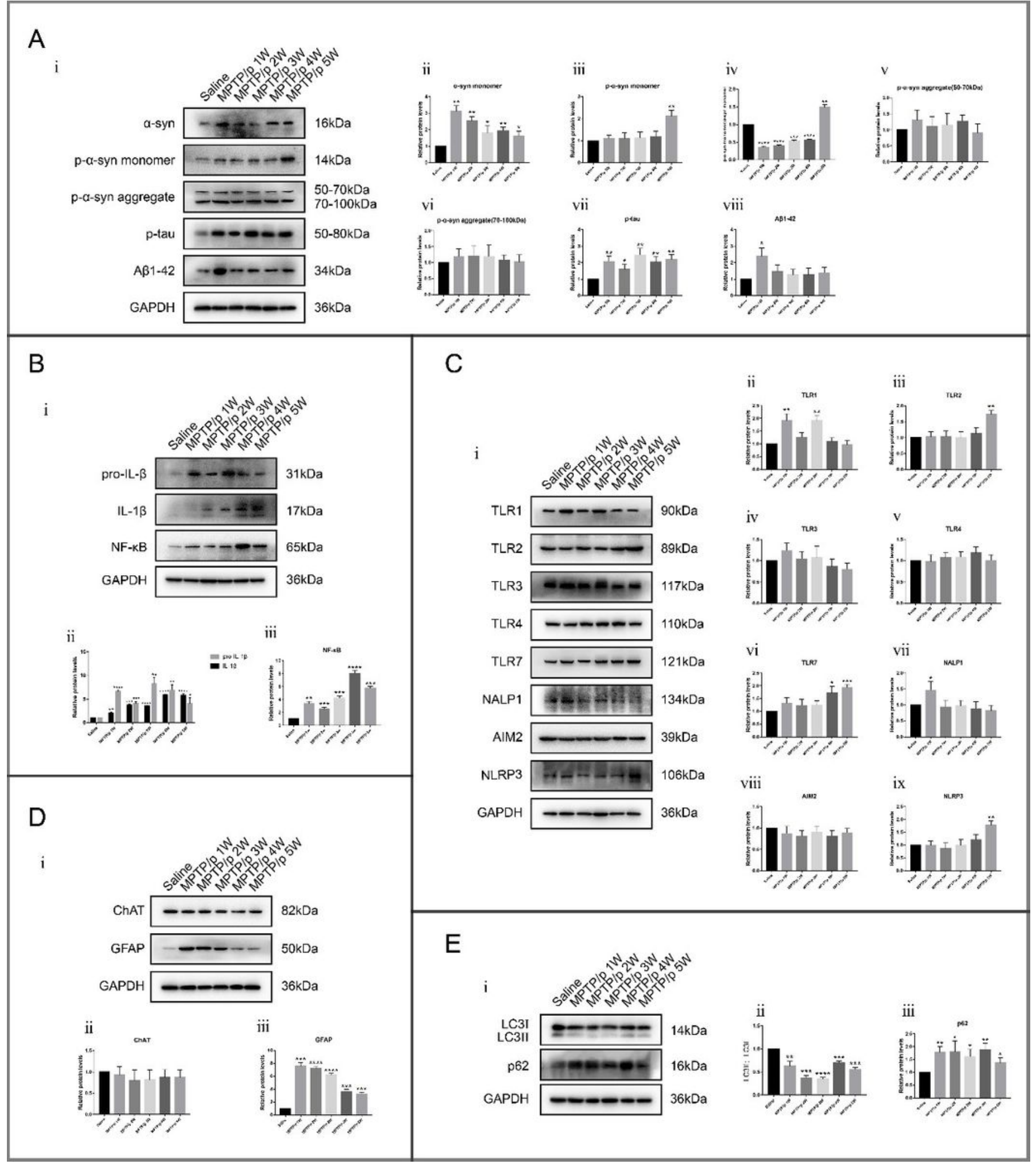

Figure 2 
WB results of various indicators in the hippocampal extracts of mice in the Saline group and MPTP/p groups in each week. i: WB band of each index. An additional movie file shows this in more detail; ii-邓: quantitative analysis of the protein expression level of the corresponding index in i. Use GAPDH as an internal reference. The data graph is plotted as Mean $\pm S D, n=4$. The statistical analysis method is t-test. * means $p<0.05$ compared with Saline group; ** means $p<0.01$ compared with Saline group; *** means $p<0.001$ compared with Saline group; and $* \star \star \star$ means $p<0.0001$ compared with Saline group. A: $a-s y n$ monomer, p-a-syn monomer and aggregate, $p$-tau and A $\beta 1-42$; B: pro-IL-1 $\beta$, IL-1 $\beta$ and NF-KB; C: TLR1, TLR2, TLR3, TLR4, TLR7, NALP1, AIM2 and NLRP3; D: ChAT and GFAP; E: LC3囚, LC3囚 and p62. 


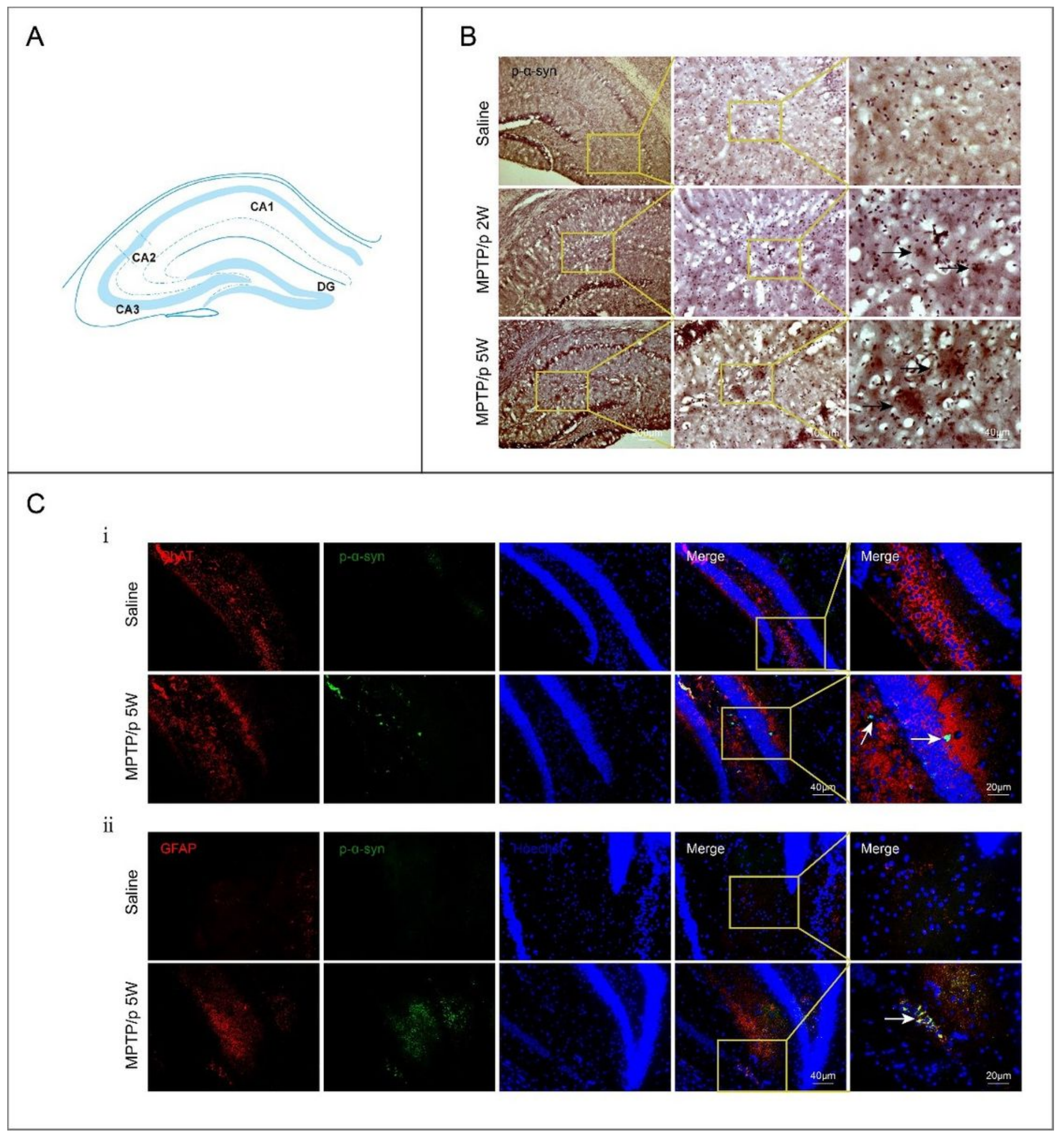

\section{Figure 3}

A: anatomical structure diagram of the mouse hippocampus. DG: denate gyrus; $B$ : the hippocampal p-asyn IHC results of mice in the Saline group and MPTP/p group at the 2 nd and 5 th week. As shown in the scale bar, the appropriate magnification is selected. The brown part shown by the black arrow is positive staining; C: IF of the mouse hippocampus in the Saline group and MPTP/p group at the 5th week, as shown in the scale bar, with appropriate magnification selected. The orange part shown by the white 
arrow is positive staining; i: ChAT and p-a-syn IF colocalization staining; ii: GFAP and p-a-syn IF colocalization staining.

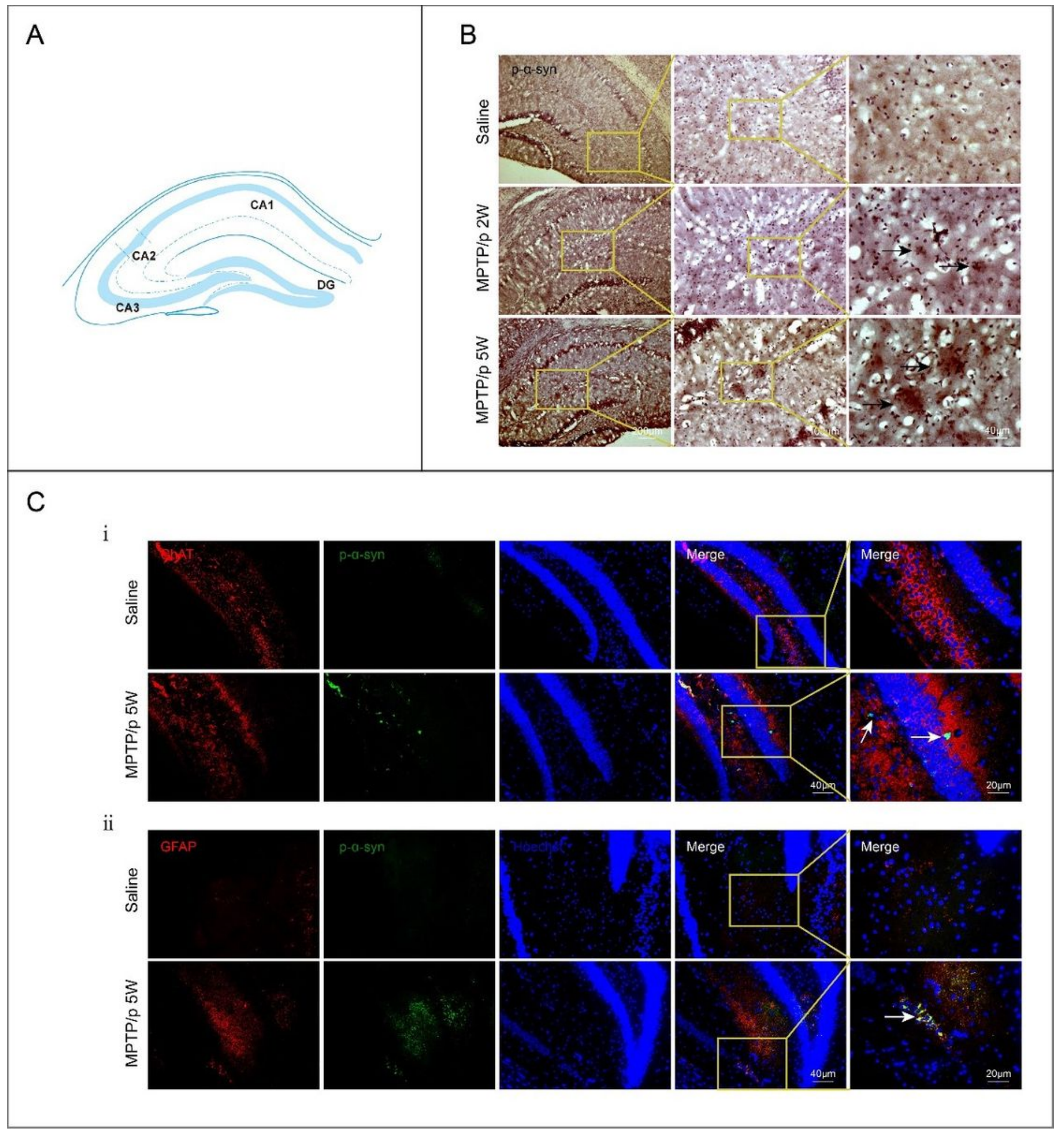

\section{Figure 3}

A: anatomical structure diagram of the mouse hippocampus. DG: denate gyrus; B: the hippocampal p-asyn IHC results of mice in the Saline group and MPTP/p group at the 2 nd and 5th week. As shown in the scale bar, the appropriate magnification is selected. The brown part shown by the black arrow is positive 
staining; C: IF of the mouse hippocampus in the Saline group and MPTP/p group at the 5th week, as shown in the scale bar, with appropriate magnification selected. The orange part shown by the white arrow is positive staining; i: ChAT and p-a-syn IF colocalization staining; ii: GFAP and p-a-syn IF colocalization staining.

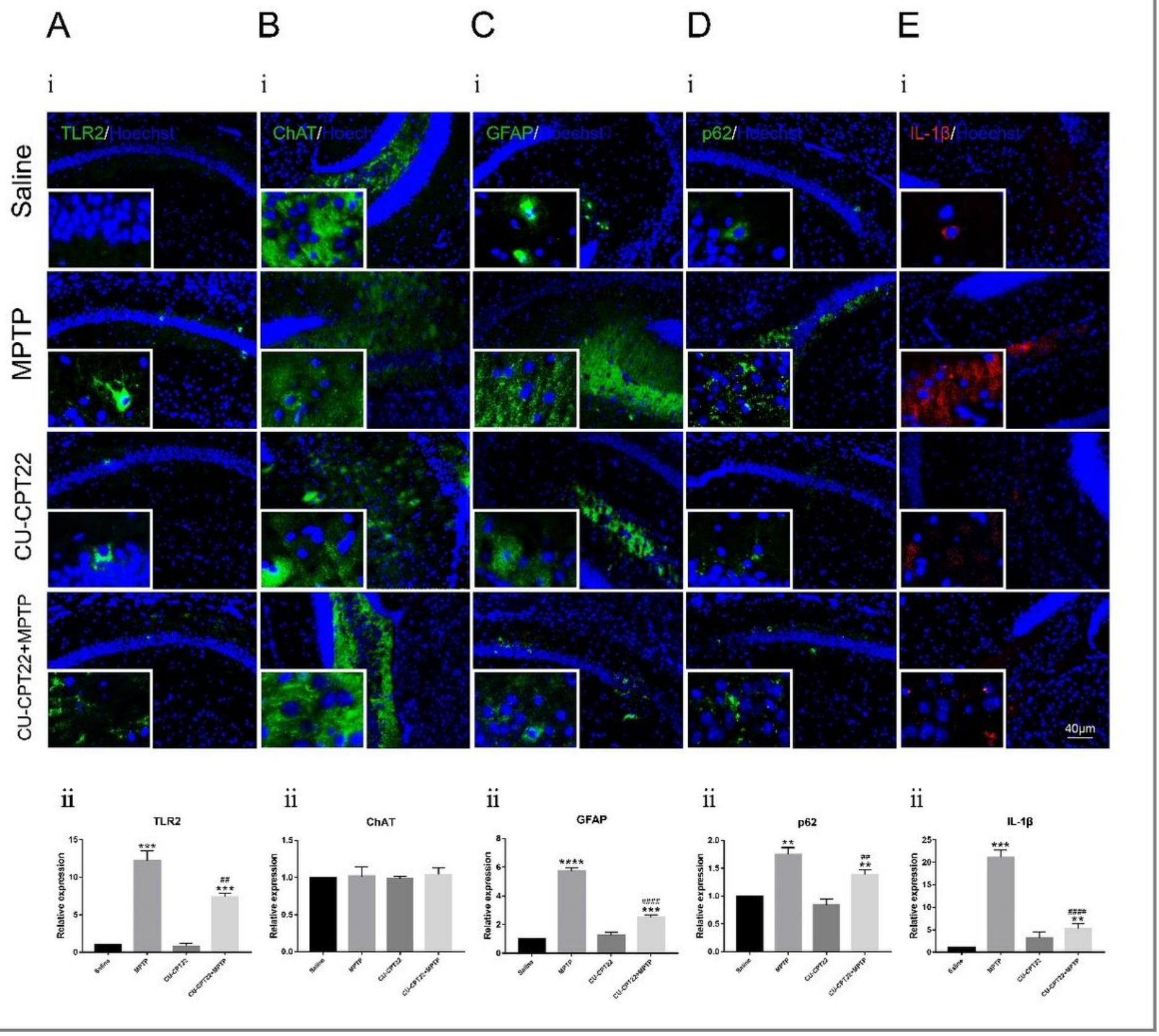

Figure 4

IF of various indexes of the mouse hippocampus in the Saline group, MPTP subacute model group, CUCPT22 group and CU-CPT22+MPTP subacute model group. Label the nucleus with Hoechst. As shown in the scale bar, the appropriate magnification is selected. i: staining of each index in the hippocampal tissue sections of each group of mice and the bottom left corner is a partial enlarged view; ii: semiquantitative analysis of the staining of the corresponding tissue sections in i. The data graph is plotted 
as Mean $\pm S D, n=6$. The statistical analysis method is t-test. ** means $p<0.01$ compared with Saline group; $* \star \star$ means $p<0.001$ compared with Saline group; $* \star \star \star ~ m e a n s ~ p<0.0001$ compared with Saline group; \#\# means $\mathrm{P}<0.01$ compared with MPTP group; \#\#\#\# means $p<0.0001$ compared with the MPTP group. The A-E indicators are: TLR2, ChAT, GFAP, p62 and IL-1 $\beta$.

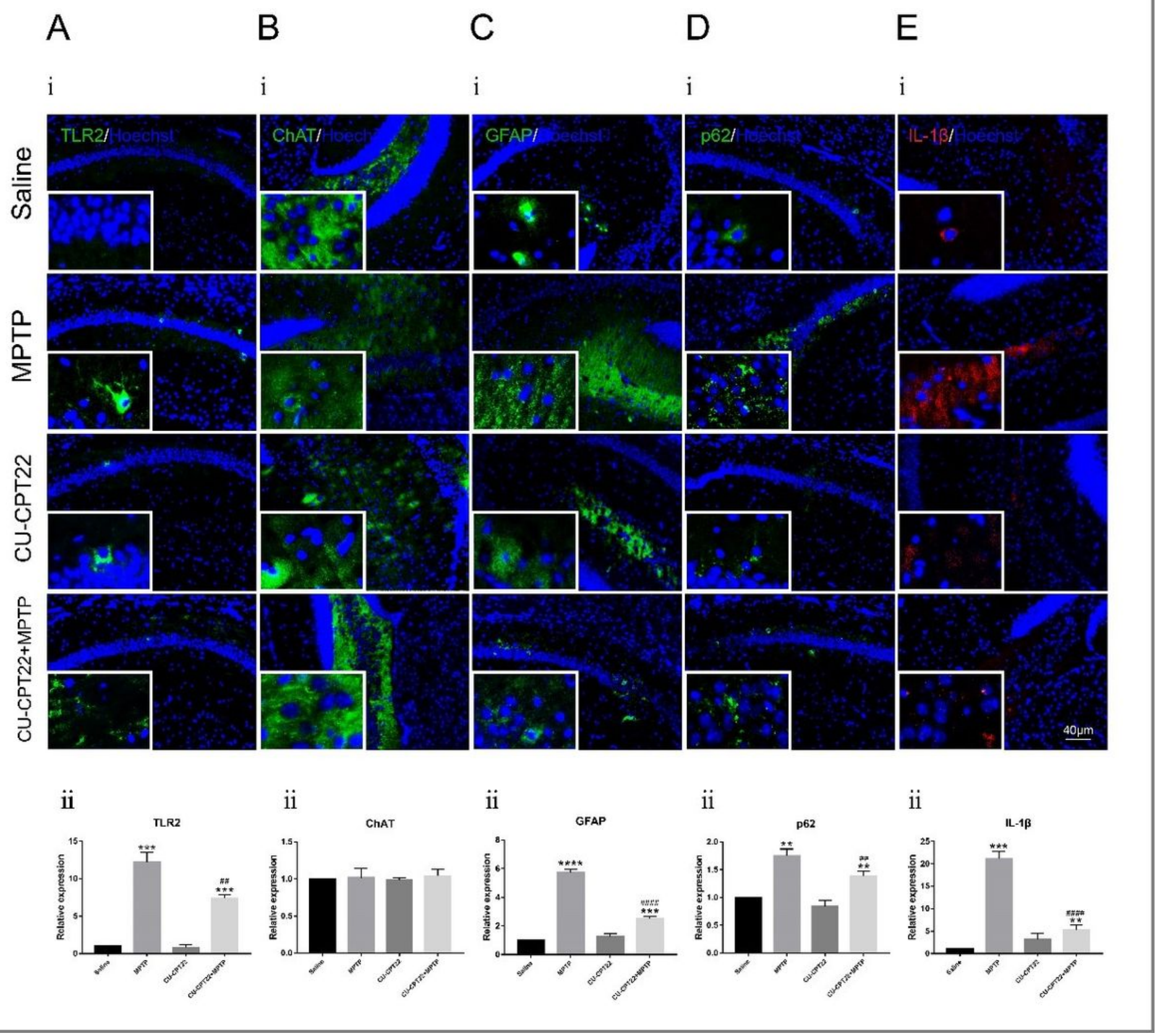

Figure 4

IF of various indexes of the mouse hippocampus in the Saline group, MPTP subacute model group, CUCPT22 group and CU-CPT22+MPTP subacute model group. Label the nucleus with Hoechst. As shown in the scale bar, the appropriate magnification is selected. i: staining of each index in the hippocampal tissue sections of each group of mice and the bottom left corner is a partial enlarged view; ii: semiquantitative analysis of the staining of the corresponding tissue sections in $\mathrm{i}$. The data graph is plotted 
as Mean $\pm S D, n=6$. The statistical analysis method is t-test. ** means $p<0.01$ compared with Saline group; $* \star \star$ means $p<0.001$ compared with Saline group; $* \star \star \star ~ m e a n s ~ p<0.0001$ compared with Saline group; \#\# means $P<0.01$ compared with MPTP group; \#\#\#\# means $p<0.0001$ compared with the MPTP group. The A-E indicators are: TLR2, ChAT, GFAP, p62 and IL-1 $\beta$.

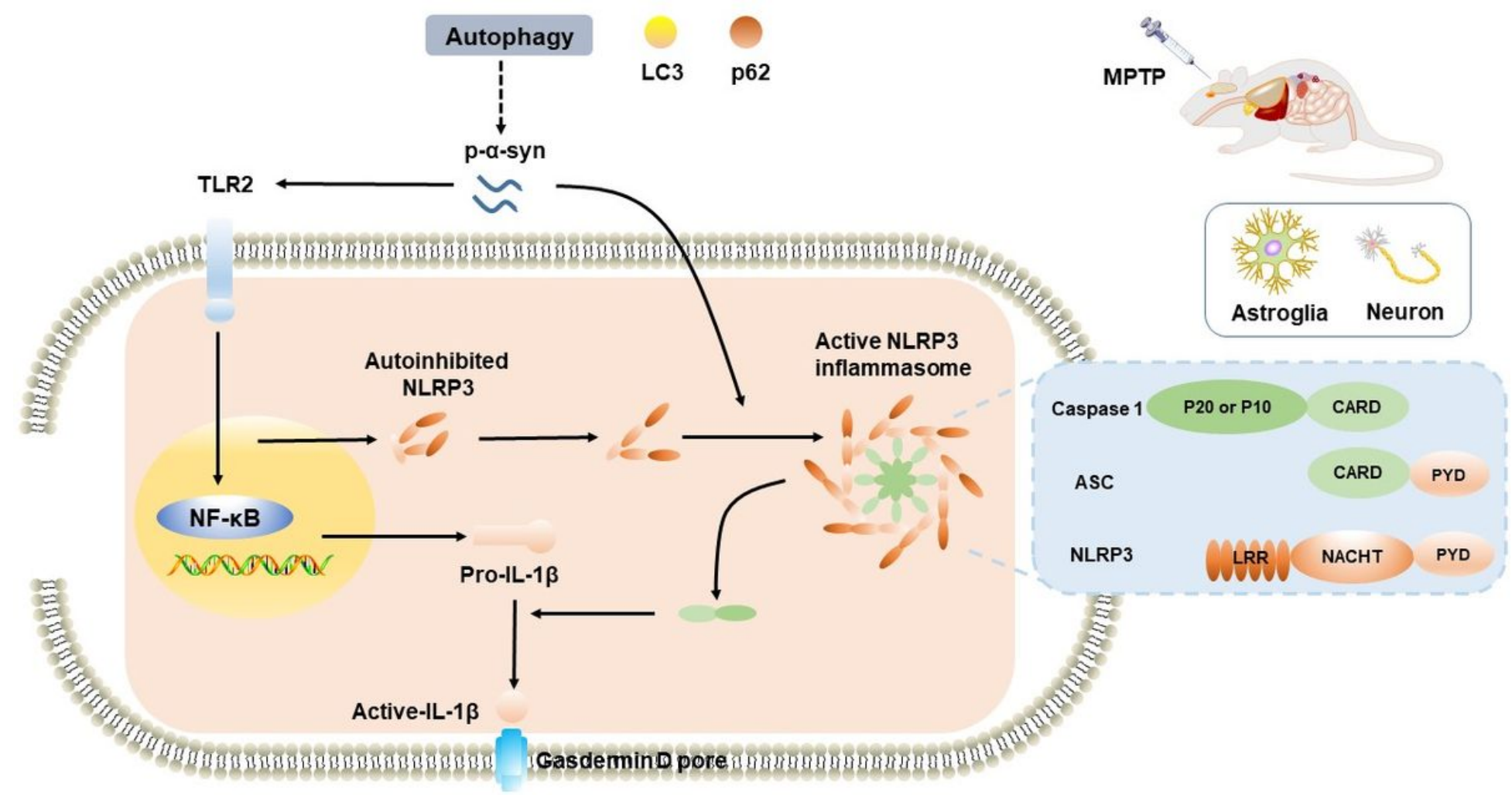

Figure 5

Hypothesis diagram. Factors such as MPTP can cause abnormal deposition of p-a-syn in the hippocampus of patients with early PD, which may be related to autophagy inhibition. TLR2 in astroglias can recognize $\mathrm{p}-\alpha-\mathrm{syn}$, activate the NF-KB pathway, and produce IL-1 $\beta$ and other inflammatory factors precursors. 


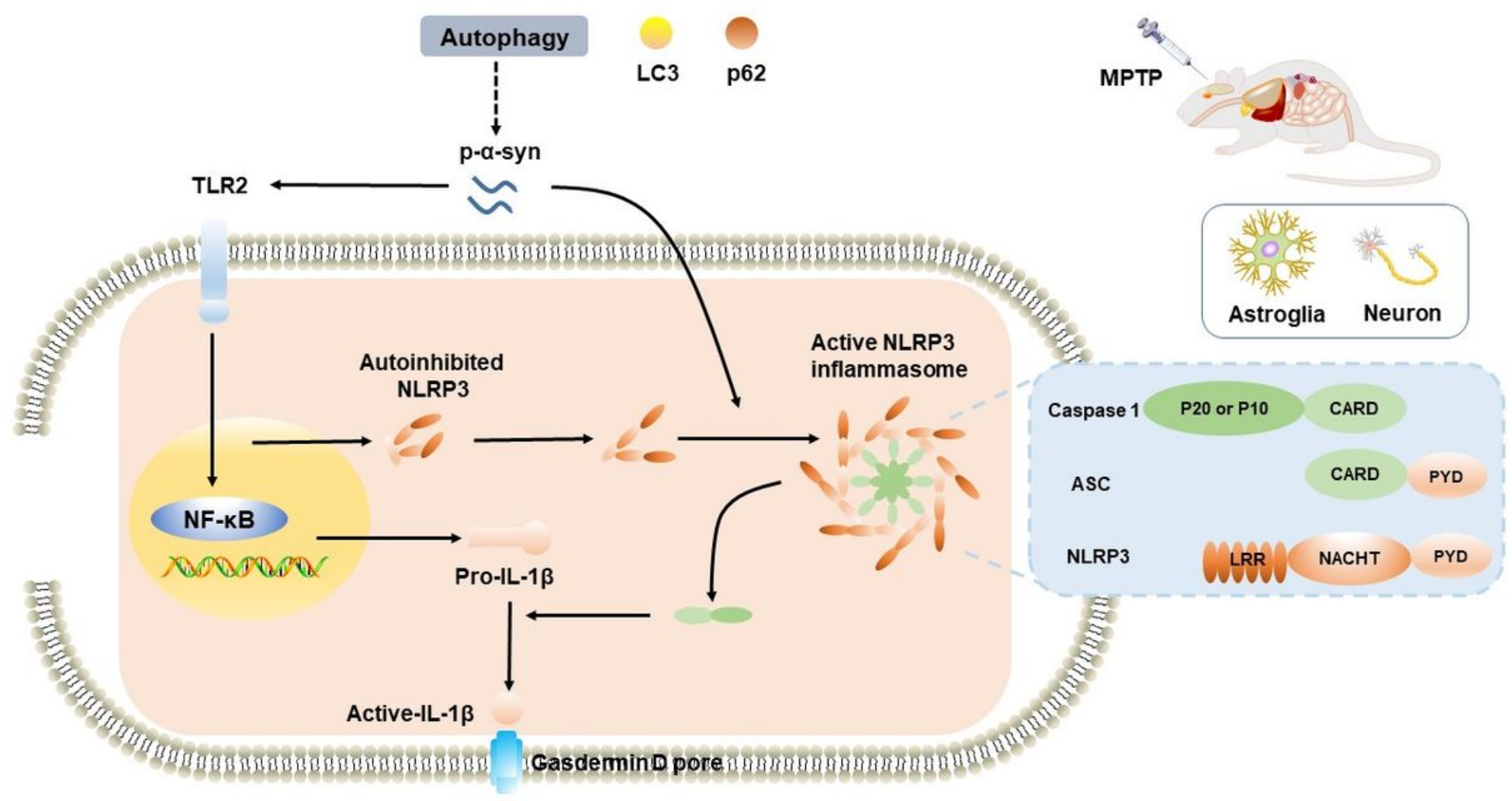

\section{Figure 5}

Hypothesis diagram. Factors such as MPTP can cause abnormal deposition of p-a-syn in the hippocampus of patients with early PD, which may be related to autophagy inhibition. TLR2 in astroglias can recognize p-a-syn, activate the NF-KB pathway, and produce IL-1 $\beta$ and other inflammatory factors precursors. 